\title{
Good Things come in all Shapes and Sizes: Meeting the Needs of People with Disabilities in Rural Georgia
}

\author{
Knicole Lee* \\ College of Nursing and Health Sciences, Valdosta State University, Georgia, USA
}

*Corresponding author: Knicole Lee, College of Nursing and Health Sciences, Valdosta State University, Georgia, USA.
Received Date: November 08, 2019

Published Date: December 12, 2019

\section{Conceptual Paper}

As the world evolves and technology changes so do healthcare needs for the people in it. Lack of access to healthcare has been an area of great concern to healthcare professionals for many years [1]. Children with disabilities are living longer, requiring increased services and have achieved the right to "Not be Left Behind". With these requirements comes the obligation to meet the needs of each of these children from birth throughout the life span U. S. Department of Education [2,3,4]. Special interest populations like people with disabilities and those in underserved areas have grown in deprivation of adequate services and lack of culturally sensitive holistic care. This article will demonstrate target populations of the model, its goals and how support of the conceptual framework identified can be utilized to achieve successful outcomes.

\section{Discussion}

Each day children are diagnosed with and treated for a vast range of disabilities. Attention is given to the immediate goal of improving health status, academic performance or developing a plan of care to promote positive behaviors and social interaction. Health professionals in rural areas are depended upon to provide quality care to patients with many diverse and specific healthcare needs. An important component of this is holistic intervention which looks at the child's socioeconomic status, cultural needs and developmental challenges that may affect the child and or the family in day to day living.

Getting to know the patients, their families, where they live and how they survive in day to day society is a major component of the framework. Being faced daily with how to meet the needs of their clients in the most cost effective, culturally sensitive and holistic manner possible. Knowing that lower economic status provides for less available resources for the home to have as well as having less ability to have access to advanced interventions that may be available to better meet the needs of the patient with a disability. Ultimately, understanding that with lower socioeconomic status comes increased rates of child abuse and neglect, so that the health professional can better educate the care coordination team to be very evaluative of the needs of each of the patients most at risk no matter what the setting. Often missing from intervention resources and care management plans is a component for transition into adulthood and successful achievement of life goals.

It is apparent that there is a need for more specific services for people with disabilities in adulthood in the areas of transition, employment, relationships and education. Not only do they need support and intervention, but their families and peers need support as well. Having a disability in some cultures is deemed as a curse or punishment [5]. Utilizing the knowledge that a holistic method is the best approach to reach the desired goal, educations and interventions should encompass a community and family portion to fully meet the needs of the total support care network.

Targeting specific populations that are at the greatest risk utilizing strong clinical knowledge to build specific interventional methods are needed to produce successful outcomes [6,7]. Designed as a vision of an APRN during graduate studies, striving to more effectively meet the needs of clients in underserved areas of rural Georgia, particularly those with disabilities across the lifespan, the utilization of the NICKELS Health Model (Nurturing Providers, Implementing Primary Rural Health Services, Caring for Kids and Adults, Empowering and Educating for Positive, Lifestyle Behaviors and Changes to Strive for Better Rural Health Across the Lifespan) will support change and care improvement for those at greatest risk. 
The NICKELS Health Model will utilize clinical expertise to target the populations in greatest need and evaluate areas of greatest deficit to design a solution. By taking ownership in each patient's care, the partners in the NICKELS Health Model demonstrate that each person is equally as important as the other. While providing this holistic and culturally sensitive care the partners will be internally processing the needs of the client population at a more broad level (Figure 1).

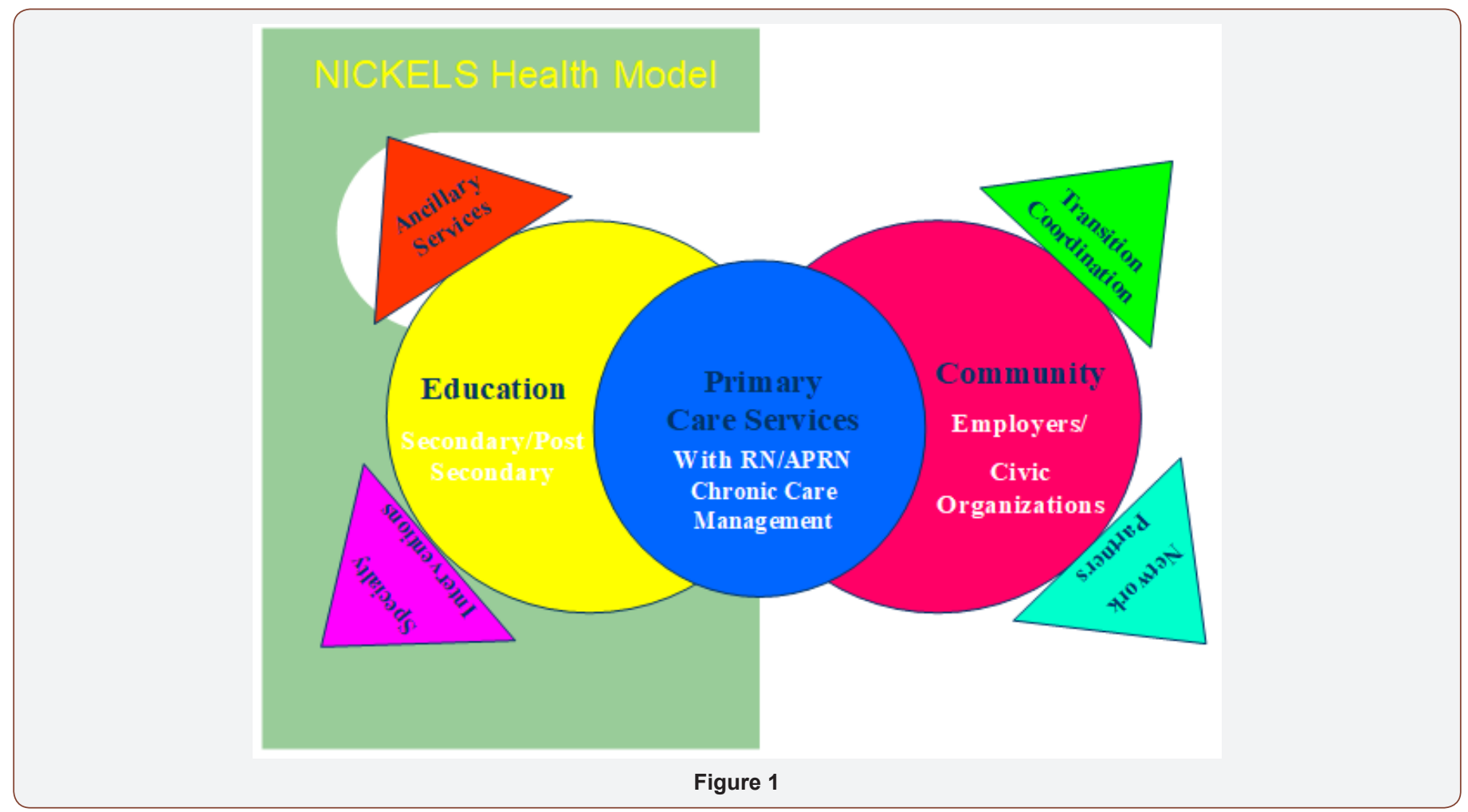

Target populations identified in the initial assessment are people with disabilities and those in underserved areas, particularly rural health. In the Improving Head Start Act of 2007, Congress notes that a minimum ten percent of slots for head start programs should go to children with disabilities [8]. According to Census 2000 and HPSA reports, target populations such as mental health are grossly underserved, thus confirming the need for the specific project focus on in this area. Healthy People 2000 and 2010 note that lack of access to healthcare, in particular mental health, to be a major area of lack of adequate service. Implementing primary rural health services through a comprehensive health plan will be the initial component for each patient served. This model of health service provides for the client to have not only provision of primary care, but management of all components of services needed to fulfill the clients' overall individual needs.

Kids and adults across the lifespan have diverse and unique healthcare needs. People with disabilities in rural areas must travel to specialty providers, have little community support and decreased access to interventional family services to help meet their stressful and exhausting needs [9]. Educating healthcare partners regarding both cultural and clinical considerations specific to the patients needs will exhibit caring at a much more individual and personal level.

Rural areas lack enough providers for primary care to the general population of patients. Providers are overwhelmed and don't always have time to address special needs of complex patients. Lack of communication amongst supporting partners for patients' total care leaves many kids and adults lacking the holistic patient care that they desperately need. Transition planning for people with disabilities nearing the age of majority is an important component of care Meese. Support is needed for electronic medical records and improved technology at primary care sites is important to facilitate the collaborative knowledge of patient needs and concerns.

Children in foster care and seasonal farm workers may live in a different town each week. As patients with disabilities exit secondary educational programs and plan for potential postsecondary education the provider must aid the patient to advocate for themselves and declare their disability so that they may receive appropriate interventional services [10]. Designing management models for seamless service provision with electronic medical records and unique coding design so that no matter where the patient receives service the provider of record will know who to contact and what type of services make up the total patient package is a model goal.

Partnerships should be made with local school systems to have a working relationship for secondary education performance, any successful interventions and access to the transition plan for the patient that was designed prior to exiting the system. For employers the vision is to become more interested in educating their managers and staff on how to meet the needs of their employees more effectively so that they are successful in their jobs. Post-secondary educators need to be empowered to meet both the content knowledge and specific learning needs of each adult learner in the classroom. Utilizing the knowledge that a holistic 
method is the best approach to reach the desired goals, education and interventions should encompass a community and family to fully meet the needs of each person with a disability across the lifespan.

\section{Summary}

Harkness, Super [11] note that supporting the daily lifestyle of the family structure while promoting positive change is key to the participants actually taking ownership of the newfound concept and utilizing it to make positive change in their individual family situations. Demonstrating to partners in the collaborative the ability to decrease expenses and increase revenue through grants, decreased inappropriate use of services and patient attendance to regular medical appointments and educational activities aids to concrete the foundation of how the NICKELS Health Model can provide success for all. As success builds in the model implementation expansion to a multi-county focus area will be utilized to aid in meeting the needs of a larger population in need.

Striving for better rural health across the lifespan is the ultimate goal of this model. Building partnerships, designing interventional programs and utilizing resources to meet the needs of underserved populations will aid to build a healthier, more successful healthcare system. Expression of cultural sensitivity as an important component of the NICKELS Health Model, it demonstrates the desire to truly meet the individual needs of each and every patient. The advanced knowledge of the participating partners makes the NICEKLS Health Model perfect to take on the challenges of healthcare today in rural Georgia. Providing foundational support, guiding program implementation and evaluating success of interventions to adapt to meet the ever-changing needs of the patient population at hand will be integral in the success of the model.

\section{Acknowledgement}

None.

\section{Conflict of interest}

No Conflict of interest.

\section{References}

1. Healthy People 2010, USA.

2. (2008) Georgia Department of Education, 2008. 2007-2008 Special Education Annual Report, USA.

3. (2008) U S Department of Education. No child left behind, USA.

4. (2008) U S Department of Education. Building the Legacy: IDEA 2004, USA

5. Andrews M M, Boyle J S (2003) Transcultural concepts in nursing care. ( $4^{\text {th }}$ edn), Philadelphia, PA, Lippincott, Williams \& Wilkins.

6. Barker AM (2009) Advanced practice nursing, essential knowledge for the profession. Sudbury, PA: Jones and Bartlett.

7. Hamric AB, Spross JA, Hanson CM (2009) Advanced practice nursing, an integrative approach. St. Louis, MS: Saunders.

8. Cortellia C (2007) Congress updates head start: new law seeks to bolster requirements for enrollment of children with disabilities. EP Magazine.

9. Evans S, Timmins B (2006) Developing coordinated, multimodal, schoolbased treatment for young adolescents with ADHD. Education and Treatment of Children 29(2): 359-378.

10. Reese RL (2001) Teaching learners with mild disabilities: Integrating research and practice. Wadsworth/Thompson Learning, USA.

11. Harkness S, Supper CM (2007) Culture and the construction of habits in daily life: implications for successful development of children with disabilities. OJTR: Occupation Participation and Health. 